Breast Cancer Research and Treatment21 December 2017, Pages 1-2

\title{
Comments on "Risk of mortality of node-negative, ER/PR/HER2 breast cancer subtypes in T1, T2, and T3 tumors" by Parise CA and Caggiano V, Breast Cancer Res Treat, 2017.
}

\section{( 哩 Article in press ? )}

- Pichilingue-Febres, A.F.Email Author,

- Arias-Linares, M.A.,

- Araujo-Castillo, R.V.

- View Correspondence (jump link)

- Escuela de Medicina, Universidad Peruana de Ciencias Aplicadas, Lima, Peru

\begin{abstract}
Author keywords

- Breast neoplasms

- Ki-67 Antigen

- Receptor, ErbB-2

- Survival analysis
\end{abstract}

We would like to express our opinion regarding a Parise and Caggiano paper recently published in your journal. We certainly believe this is a great contribution, since it found that node-negative HER2 (+) breast cancer patients have better survival contrary to the common knowledge. This finding could reflect the consequences of targeted therapies that are changing the natural history of the disease. However, we think that such an interesting analysis could also have been done with stage III and IV patients, since this group of people could benefit greatly from these findings. In fact, new guidelines now recommend the use of HER2specific therapy for stage IV patients with positive markers, even for life if they do not show signs of progression. Additionally, we would like to discuss the value of adding the Ki-67 marker to the classification proposed by the authors, because several papers consider it an important prognostic factor. (c) 2017 Springer Science+Business Media, LLC, part of Springer Nature 
- ISSN: 01676806

- CODEN: BCTRD

- Source Type: Journal

- Original language: English

- DOI: $10.1007 / \mathrm{s} 10549-017-4620-y$

- Document Type: Article in Press

- Publisher: Springer New York LLC 